FTPI-MINN-13/33, UMN-TH-3304/13

\title{
New and Old about Renormalons: In Memoriam Kolya Uraltsev日
}

\author{
M. Shifman \\ William I. Fine Theoretical Physics Institute, University of Minnesota, \\ Minneapolis, MN 55455, USA
}

\begin{abstract}
I summarize what we know of renormalons from the 1970s and 80s: their uses and theoretical status. It is emphasized that renormalons in QCD are closely related to the Wilsonean operator product expansion (OPE) - a setup ideally suited for dealing with the factorially divergent series reflecting infrared dynamics. I discuss a breakthrough proposal due to Uraltsev et al. to use renormalons to evaluate nonperturbative (power) corrections in the processes without OPE. Some fresh ideas which were put forward recently are briefly discussed too, with emphasis on a possible relationship between resurgence via trans-series and OPE.

This article is devoted to the memory of my friend Kolya Uraltsev. I should emphasize that these are my personal recollections. Other people who closely knew Kolya may or may not agree with my opinions.
\end{abstract}

${ }^{1}$ To be published in Nikolai Uraltsev Memorial Volume, (World Scientific, Singapore, 2014). 


\section{Foreword:}

\section{Nikolai (Kolya) Uraltsev}

Kolya, Alexei Anselm's student, was one of the most prominent theorists from the young generation of the Gribov Leningrad school. The heavy quark theory acquired the level of perfection it enjoys now to a large extent due to his works on quantum chromodynamics. In this area, there was no higher authority in the world than Kolya.

In science Kolya was a "slowpoke," in the sense that each new result or new assertion in his field - the theory of heavy quarks - had to be critically processed before being accepted (or not). Coming across something new Kolya pondered on all sides of this "something new" with incredible diligence. There was no way any ambiguity could be left after Kolya. He almost physically suffered from sloppy works and light-minded authors. Kolya gave no quarter to such speakers at seminars or conferences, no matter what regalia they may have possessed. With them he was aggressive and restless until he had exposed all loopholes in the arguments. This "inconvenient" style - science above everything else - that Kolya had learned from Gribov, he carried through his life, without changing it in the West, where it (the style) is almost extinct due to incompatibility with political correctness. Frankly speaking, physicists from the West slightly feared Kolya. None of the heavy quark theorists wanted to be ground by "millstones" in Kolya's mind.

It is ironic that in everyday life Kolya was not only shy, but rather supershy. You can hardly find such shy people nowadays. For him it was a problem to talk to a stranger or to respond to the harsh words of an insolent fellow.

Every summer Kolya went on archaeological excavations at the Kola Peninsula. There he met his future wife. Well ... this statement is not entirely accurate. Lilya (that's her name) at the time worked in Leningrad's Hermitage and also used to go to Kola Peninsula excavations. Once she told me: "Kolya stared at me for a long time, but did not dare to approach. Then I realized that if I do not take matters into my own hands, we will return to Leningrad without getting acquainted ..."

When Kolya was thinking about physics, he did not notice anything around him. Once during a conference, after a session, we walked out of the conference hall to the street under heavy rain. Everybody opened umbrellas right away. Kolya did not react to a change in the environment from 
comfortable to dramatically uncomfortable, and continued the discussion as if nothing had happened ... He kept a clean child's soul.

We - Kolya and I - published 17 joint works: the first in 1987 and the last in 1998. Especially productive was our collaboration during the academic year 1994/95 when Kolya spent the whole year with us at the University of Minnesota. Many ideas conceived during this year became parts of subsequent research on heavy quark theory. Here I would like to single out a particularly exciting insight: the use of renormalons as a tool for revealing power terms in the processes without the operator product expansion.

Kolya could repair with his own hands any damage to any vehicle, including those most modern and stuffed with electronics. It was his passionate hobby. In 1996, we spent six months together at CERN. For everyday commuting I bought a used Audi, which had problems all the time. In the Swiss garages they asked from me exorbitant prices for repairs. Kolya coped effortlessly.

In fact, Kolya could fix just about anything, not only cars. In this, like in physics, he was inquisitive; he loved the process of learning "how things work," be it a $B$-meson decay or a leak in a boat.

Striking thoroughness - that's how I would characterize Kolya's approach to every aspect of his life and work. During his 30-year career in theoretical physics Kolya closely and productively interacted with many colleagues on three continents, St. Petersburg, CERN, Technion, Milan, Orsay, FTPI, University of Notre Dame, and University of Siegen. I am sure that's how they will remember him - a deep thinker and a reliable friend.

Death is always untimely. When Kolya's heart stopped on February 13, 2013 he was only 54 years old, full of plans for the future both in science and life. Even now, six months after his tragic death, it is not easy for me to write this in memoriam article in a logically-ordered manner. Apparently, I will have to settle for less.

\section{Introduction}

One can say that Kolya burst onto heavy quark theory like a meteor. Our first (occasional) scientific encounter occurred in 1986 [1]. Shortly after, our paths departed: he delved in the problem of $C P$ violation for six long years [2, 3], while I returned to nonperturbative supersymmetry [4]. I was still heavily 
involved in this topic when Kolya appeared in our Institute 2 in 1992 full of enthusiasm with regards to a consistent theory of $1 / m_{Q}$ expansion in heavy quarks based on the operator product expansion (OPE). Elements of this theory already existed [5, 6, 7]. However, they represented general guidelines rather than a theoretical construction worked out in detail. Applications were rather scarce. Kolya's enthusiasm was contagious, and shortly after both, Arkady Vainshtein and myself, got fully involved. One of the most elegant results established by Kolya and collaborators [8] (see also [9]) was the absence of the $1 / m_{Q}$ correction in the total inclusive decay widths of the heavy-flavor hadrons. This theorem (sometimes referred to as the CGG BUV theorem) made its way into textbooks, let alone its practical importance for precision determination of $V_{c b}$ and $V_{u b}$ from data.

In twenty years that elapsed after 1992 Kolya managed to make definitive contributions to many topics from the heavy quark theory. It is fair to say that he left no stone unturned. His imprint is seen everywhere. Needless to say, I will be unable to cover all these topics. Instead, I will focus on one particular topic - determination of the power of $1 / m_{Q}$ (or $1 / Q$ ) terms from renormalons in the processes without OPE - in which Kolya was a trailblazer 3

\section{Pioneers}

Two papers, [15] and [16], which appeared on ArXiv on the same day, were the first to suggest the usage of renormalons for indication of the power of nonperturbative corrections (i.e. $1 / Q$ or $1 / m_{Q}$, or squares, cubes etc. of the above parameters) in the processes without OPE.

The next relevant paper was [17], where the idea was first applied to hadronic event shapes.

Before explaining how this works, I will have to remind you what renormalon is. To this end I will have to start from the factorial divergences of the perturbative series.

\footnotetext{
${ }^{2}$ William I. Fine Theoretical Physics Institute, University of Minnesota.

${ }^{3}$ The reader unfamiliar with the range of questions associated with OPE, $1 / m_{Q}$ corrections in heavy quark theory, renormalons and all that is advised to turn to reviews [10, 11, 12, 13, 14].
} 


\section{Dyson argument and factorial divergences}

Sixty two years ago Freeman Dyson completed his famous paper entitled "Divergences of Perturbation Theory in Quantum Electrodynamics" [18] (reprinted in [19]). He argued that the series in $e^{2}$ in QED could not be convergent due to the fact that analytic continuation to negative $e^{2}$ produced a theory with unstable vacuum. This became known as the Dyson argument. Shortly after, Thirring evaluated [20] the number of diagrams in $\lambda \phi^{3}$ field theory in high orders and came to the conclusion that the perturbative series in this theory is factorially divergent. In 1977 various field theories, including $\lambda \phi^{4}$, were thoroughly studied by Lipatov [21] who came to the same general conclusion: the perturbative series are asymptotic and characterized by the factorial divergence of the form

$$
Z=\sum_{k} C_{k} \alpha^{k} k^{b-1} A^{-k} k !
$$

This is reviewed in some detail e.g. in [19]. The notation in Eq. (1) is as follows: $\alpha$ is the expansion parameter $4 k$ is the number of loops, $C_{k}$ 's are numerical coefficients of order one, and $b$ and $A$ are numbers.

Arkady Vainshtein was the first to point out [23] (see [24]) that the factorial divergence in (1) is in one-to-one correspondence with the probability of the under-the-barrier penetration (vacuum instability in field theory language) for unphysical - negative - values of the expansion parameter. Ten years later this relation was rediscovered by Bender and $\mathrm{Wu}[25]$ in the quartic anharmonic oscillator or, which is the same, in $\lambda \phi^{4}$ theory.

The factorial divergence of the perturbative series discussed in [18, 19, 23, 24, 25] can be traced back to the factorially large number of multiloop Feynman diagrams (i.e. $k \gg 1$ ).

Renormalons which we will focus later have nothing to do with this mechanism. As was noted in [26], there exists a class of isolated graphs, in which each diagram grows factorially as we increase the number of loops. It is these graphs that are called renormalons. The theoretical feature responsible for the renormalon factorial divergence (11) is the logarithmic running of the effective coupling constant.

\footnotetext{
${ }^{4}$ In QED it is customary to define $\alpha \equiv e^{2} /(4 \pi)$. The asymptotic divergence of the coefficients in QED is somewhat more contrived [22] than in (11) due to the fact that the QED loops are due to fermions.
} 


\section{Borel summability}

Instead of the asymptotic series (1) let us introduce the Borel transform

$$
B_{Z}(\alpha)=\sum_{k} C_{k} \alpha^{k} k^{b-1} A^{-k}
$$

In Eq. (22) the $k$-th term of expansion (11) is divided by $k$ !, which implies, in turn, that the singularity of $B_{Z}(\alpha)$ closest to the origin in the $\alpha$ plain is at distance $A$ from the origin. Thus, the sum (2) is convergent.

Mathematicians would say that the function defined by (2) is obtained from (1) by the inverse Laplace transformation.

It is quite obvious that one can recover the original function $Z$ performing the following integral transformation (the Laplace transformation):

$$
Z(\alpha)=\int_{0}^{\infty} d t e^{-t} B_{Z}(\alpha t)
$$

see e.g. [27], Sect. 37.3. The integral representation (3) is well-defined provided that $B_{Z}(\alpha)$ has no singularities on the real positive semi-axis in the complex $\alpha$ plane. This is the case if the asymptotic series (11) is signalternating, $C_{k} \sim(-1)^{k}$, (and then so is (2) ). If $B_{Z}(\alpha)$ has singularities on the real positive semi-axis (as is the case if the coefficients $C_{k}$ are all positive, or all negative), then the integral (3) becomes ambiguous. The ambiguity is of the order of $e^{-A / \alpha}$. One cannot resolve this ambiguity on the basis of purely mathematical arguments. More information is needed, which can be provided only by underlying physics.

In problems at weak coupling additional physical information can be obtained by quasiclassical methods. Indeed, at weak coupling deviations from perturbation theory are due to classical solutions with nonvanishing action, such as instantons or instanton-antiinstanton (IA) pairs. Say, in the quantal problem of the double-well potential, the contribution of the instantonantiinstanton pair is ambiguous per se. However, one can combine (3) with the latter in such a way, that in the final answer these two ambiguities cancel, giving rise to a well-defined expression [28, 29]. The next ambiguity occurs at the level of two instanton-antiinstanton pairs. It is canceled against the ambiguity in perturbation theory in the sector of a single instanton-antiinstanton pair plus a subleasing singularity [30] in (3). The process of cancellation of ambiguities is repeated ad infinitum. Continuing this procedure one arrives 
at the so-called trans-series combining perturbative and quasiclassical nonperturbative expansion at weak coupling. In a slightly simplified form the resurgence and trans-series can be expressed by the formula

$$
Z(\alpha)=\sum_{k=0}^{\infty}\left\{c_{0, k}+c_{1, k} \alpha+c_{2, k} \alpha^{2}+c_{3, k} \alpha^{3}+\ldots\right\} e^{-k A / \alpha}
$$

where for each given $k$ the coefficients $c_{n, k}$ are factorially divergent in $n$, and the sum in $n$ in the braces (for each given $k$ ) is regularized in a well-prescribed manner. I will say a few words on the nature of the $k$ series later.

In quantum mechanics the construction of the trans-series was explored in [28, 29, 30, 31, 32, 33. Recently a progress along these lines was achieved in field theory too [34, 35.

To make sure that a field-theoretical model under consideration is weakly coupled, it was analyzed [34, 35] in cylindrical geometry $R_{1} \times S_{1}(r)$, with a compactified dimension of a very small size $r$. Then, in much the same way as in the above quantal problem, it proved to be possible to identify quasiclassical field configurations responsible for nonperturbative contributions [36, 37, to be combined with the Borel-resummed perturbative series.

It is quite plausible that in weakly coupled field theories a complete resurgence can be achieved along these lines, and at least some quantities are representable in the form of trans-series combining Borel-resummed perturbation theory with a (infinite) set of nonperturbative effects derivable from quasiclassical considerations. What remains to be seen is whether this program works in a more general setting of any weakly coupled field theory, for instance, in fully Higgsed Yang-Mills theory, and if yes, in which particular way. At the moment the idea of matching the factorial divergence to quasiclassical field configurations in fully Higgsed Yang-Mills theories is barely explored 5

If this idea survives in a more general formulation, the next intriguing question is obvious: whether or not a connection to strong coupling regime can be revealed. Note that at weak coupling continuous symmetries such as the chiral symmetry cannot be spontaneously broken. Therefore, a parallel between resurgence via trans-series in quantum mechanics on the one hand and OPE in QCD and similar theories on the other, which of course comes to one's mind, cannot be complete.

\footnotetext{
${ }^{5}$ Some hypotheses are discussed in Sect. 12 .
} 


\section{The first source of factorial divergence}

In quantum mechanics the coupling constant is fixed. In Yang-Mills field theory (e.g. QCD) the very notion of the smallness of the coupling constant is meaningless, since the coupling constant depends on scale; it runs and becomes strong at momenta of the order of dynamical scale $\Lambda$. At such momenta dynamics are by no means exhausted by perturbation theory and quasiclassical nonperturbative effects. In fact, in the infrared domain, at strong coupling, both cannot even be consistently defined. Below we will discuss what can be done under the circumstances.

For a short while, let us close our eyes at this feature pretending that somehow the blow off of $\alpha_{s}$ in the infrared (IR) domain is not essential. This neglect will be corrected shortly. In Yang-Mills theory one can identify at least two sources for the factorial divergence of the perturbative series. First, the number of various Feynman graphs with $n$ loops grows as $n !$. This feature (similar to that one encounters in quantum mechanics) was known already to the explorers of QED from the times of the Dyson argument, see Sect. 3. As a result, even if each graph is of the order of unity in appropriate units, the contribution of the set of the $n$-loop graphs will be of the order of $n ! \alpha^{n}$. At $n \sim 1 / \alpha \gg 1$ multiloop graphs are typically represented by soft fields which can be viewed as quasiclassical field configurations, for instance, instantons. Instanton contributions to correlation functions are $\sim \exp \left(-\frac{2 \pi}{\alpha}\right)$.

If this were the only source, the problem could be eliminated in an elegant way, which can be traced back to 't Hooft's observation [38] that in the limit

$$
N \rightarrow \infty, \quad N \alpha \text { fixed }
$$

where $N$ is the number of colors, only planar diagrams survive. The limit (5) is referred to as the 't Hooft limit. Three years after 't Hooft's original work a remarkable theorem was proved [39]: the number of planar diagrams with $n$ loops $\nu(n)$ does not grow with $n$ factorially, rather

$$
\nu(n) \sim C^{n}, \quad n \gg 1
$$

where $C$ is a numerical constant. In one-to-one correspondence with this fact is the vanishing of the instanton contribution at $N \rightarrow \infty$. Indeed, at weak coupling in the 't Hooft limit

$$
\frac{2 \pi}{\alpha} \sim \operatorname{const} N
$$


and the instanton contribution is exponentially suppressed.

One can identify another source of the factorial divergence - unique diagrams of a special type present in Yang-Mills which produce $n$ ! not because there are many of them, but because a single graph with $n$ loops is factorially large. As was mentioned previously, such diagrams are called renormalons [26, 40]. In the subsequent section we will consider them in more detail.

\section{Renormalons}

Both, ultraviolet (UV) and IR renormalons can be seen in the bubble diagram depicted in Fig. 1, where the dashed line represents an external (vector) fermion current, the solid lines show fermion propagation while the curvy lines stand for gluons. Consider the correlation functions of two vector currents of massless quarks

$$
\begin{aligned}
\Pi_{\mu \nu}(q) & =i \int d^{4} x e^{-i q x}\left\langle T\left[j_{\mu}(x) j_{\nu}(0)\right]\right\rangle=\left(q_{\mu} q_{\nu}-q^{2} g_{\mu \nu}\right) \Pi\left(Q^{2}\right) \\
j_{\mu} & =\bar{\psi} \gamma_{\mu} \psi
\end{aligned}
$$

where $\psi$ is the quark field; we assume the number of flavors to be $N_{f}$, and denote

$$
Q^{2}=-q^{2}
$$

so that in the Euclidean domain $Q^{2}$ is positive. The number of colors $N_{c}=3$. It is convenient to analyze the Adler function defined as

$$
D\left(Q^{2}\right)=-4 \pi^{2} Q^{2} \frac{d \Pi\left(Q^{2}\right)}{d Q^{2}}
$$

and normalized to unity in the leading order. The bubble diagrams with the fermion loop insertions are gauge-invariant per se. Needless to say, in the given order there are many other graphs, but we will focus on those presented in Fig. 1, which will be sufficient for identification of renormalons.

The gauge coupling runs, and we must specify which particular coupling constant is used in the expansion. The Adler function, being expressed in terms of $\alpha_{s}(Q)$, is finite. It seems obvious that the external momentum $Q^{2}$ sets the scale of all virtual momenta in loops, and we should use $\alpha_{s}(Q)$. Is it indeed the case? 


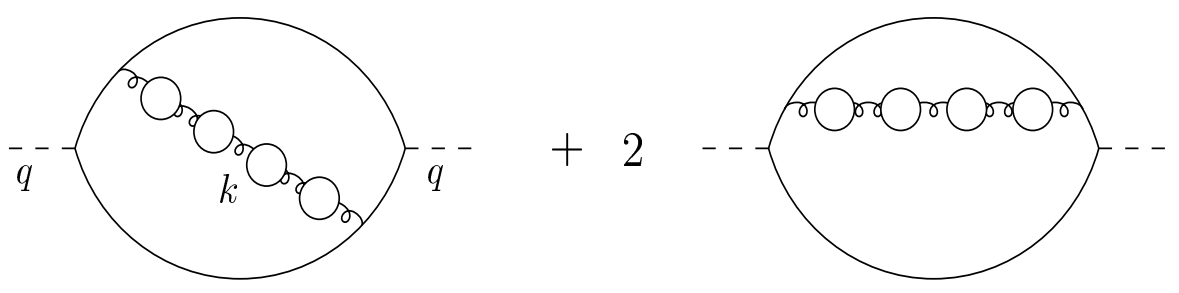

Figure 1: Bubble diagrams for the Adler function consists of all diagrams with any number of fermion loops inserted into a single gluon line. Then we replace $\beta_{0}^{f}$, the fermion contribution to the first coefficient of the $\beta$ function by the full $\beta_{0}$. This is a convenient computational device.

The answer to the above question is negative. In high orders of perturbation theory there appears an additional parameter $n$, the order of perturbation theory, which changes the naive estimate $k \sim Q$, see Fig. 1, To see that this is the case, let us have a closer look at Fig. 1 before integrating over $k$. The exact result for fixed $k^{2}$ was found by Neubert [41]. However, we will not need it since for our illustrative purposes it is sufficient to use a simplified interpolating expression [42] collecting all fermion bubble insertions 6 in the gluon propagator: $0,1,2$ and so on,

$$
D=C \times Q^{2} \int d k^{2} \frac{k^{2} \alpha_{s}\left(k^{2}\right)}{\left(k^{2}+Q^{2}\right)^{3}},
$$

which coincides with the exact expression [41] in the limits $k^{2} \ll Q^{2}$ and $k^{2} \gg Q^{2}$, up to minor irrelevant details. The coefficient $C$ in Eq. (10) is a numerical constant and $\alpha_{s}\left(k^{2}\right)$ is the running gauge coupling constant,

$$
\alpha_{s}\left(k^{2}\right)=\frac{\alpha_{s}\left(Q^{2}\right)}{1-\frac{\beta_{0} \alpha_{s}\left(Q^{2}\right)}{4 \pi} \ln \left(Q^{2} / k^{2}\right)} .
$$

The definition of the coefficients in the $\beta$ function is given in Appendix.

\footnotetext{
${ }^{6}$ The fermion bubbles in Fig. 1 produce only the fermion contribution to $\alpha_{s}\left(k^{2}\right)$ usually denoted by $\beta_{0}^{f}$. However, then we can replace $\beta_{0}^{f}$ by the full $\beta_{0}$. Note that adding the gluon and ghost bubbles is not sufficient (in particular, one would get a gauge noninvariant expression). The replacement $\beta_{0}^{f} \rightarrow \beta_{0}$ incorporates some additional contributions. Note that $\beta_{0}^{f}$ and $\beta_{0}$ have opposite signs - a crucial feature as we will see below.
} 
Now, let us examine the Adler function (10) paying special attention to the logarithmic dependence in (11), a crucial feature of QCD. We will first focus on the IR domain. Omitting the overall constant $C$, inessential for our purposes, we obtain

$$
D\left(Q^{2}\right)=\frac{1}{Q^{4}} \alpha_{s} \sum_{n=0}^{\infty}\left(\frac{\beta_{0} \alpha_{s}}{4 \pi}\right)^{n} \int d k^{2} k^{2}\left(\ln \frac{Q^{2}}{k^{2}}\right)^{n}, \quad \alpha_{s} \equiv \alpha_{s}\left(Q^{2}\right)
$$

which can be rewritten as

$$
D\left(Q^{2}\right)=\frac{\alpha_{s}}{2} \sum_{n=0}^{\infty}\left(\frac{\beta_{0} \alpha_{s}}{8 \pi}\right)^{n} \int d y y^{n} e^{-y}, \quad y=2 \ln \frac{Q^{2}}{k^{2}} .
$$

The $y$ integration in Eq. (13) represents all diagrams of the type depicted in Fig. 1 after integration over the loop momentum $k$ of the "large" fermion loop (and the angles of the gluon momentum).

The $y$ integral from zero to infinity is $n$ !. A characteristic value of $k^{2}$ saturating the integral is

$$
y \sim n \text { or } k^{2} \sim Q^{2} \exp \left(-\frac{n}{2}\right) .
$$

Thus, if $Q^{2}$ is fixed and $n$ is sufficiently large, the factorial divergence of the coefficients in (12) is indeed due to the infrared behavior in the integral (10). For what follows let us note that if at small $k^{2} \sim \Lambda^{2}$ the diagram in Fig. 1 ceases to properly represent non-Abelian dynamics (which is the case in QCD due to strong coupling in the IR), then the integral must be cut off from below at $k^{2}=\Lambda^{2}$, or at $y=n_{*}$ at large $y$. Here for each given $Q^{2}$

$$
n_{*}=2 \ln \frac{Q^{2}}{\Lambda^{2}} .
$$

The summation of factorially divergent terms in the formula

$$
D\left(Q^{2}\right)=\frac{\alpha_{s}}{2} \sum_{n=0}^{\infty}\left(\frac{\beta_{0} \alpha_{s}}{8 \pi}\right)^{n} n !
$$

ceases to be valid at $n=n_{*}$. At $n>n_{*}$ the factorial growth is suppressed, see Fig. 2, and must be truncated,

$$
D\left(Q^{2}\right) \rightarrow \frac{\alpha_{s}}{2} \sum_{n=0}^{n_{*}}\left(\frac{\beta_{0} \alpha_{s}}{8 \pi}\right)^{n} n !
$$




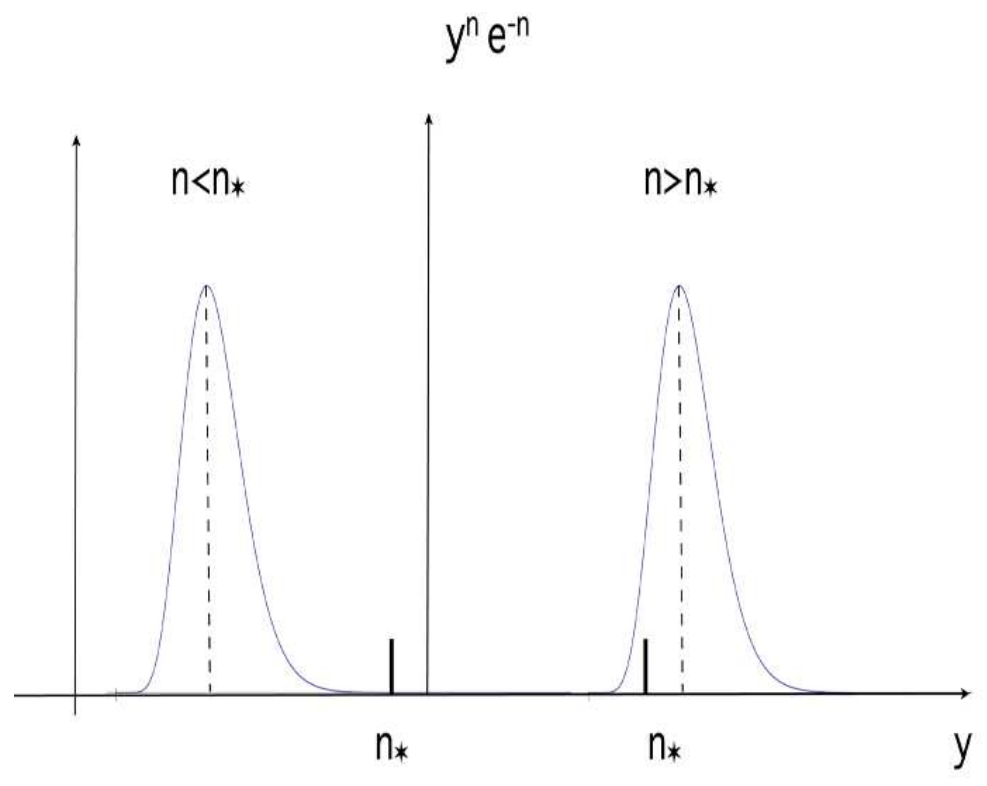

Figure 2: The plot of the integrand in Eq. (12) for two values of $n$, "small" and "large." A sharp peak at $y \sim n$ saturates the integral. In the left plot $n<n_{*}=$ $2 \ln \left(Q^{2} / \Lambda^{2}\right)$ and the forbidden domain $k^{2} \sim \Lambda^{2}$ does not contribute to the factorial factor. In the right plot $n>n_{*}$. The $y$ integration has to be cut off at $y=n_{*}$, which tempers the factorial growth.

Note that $n_{*}$ is also the critical value of the asymptotic series (16), i.e. the value at which the accuracy of approximation is the best. At $n=n_{*}$ the asymptotic series (16) achieves the highest accuracy. Truncation at $n=n_{*}$ ensures the deviation from the exact result to be $\exp \left(-\frac{8 \pi}{\beta_{0} \alpha_{s}}\right) \sim \Lambda^{4} / Q^{4}$, the same as the infrared sensitivity to the domain $k^{2} \sim \Lambda^{2}$.

All terms in (16) have the same sign, which means that the asymptotic series per se is not Borel-summable.

Now let us briefly consider the large $k^{2}$ domain in (10). At large $k^{2}$

$$
D\left(Q^{2}\right)=Q^{2} \alpha_{s} \sum_{n=0}^{\infty}\left(\frac{\beta_{0} \alpha_{s}}{4 \pi}\right)^{n}(-1)^{n} \int d k^{2} \frac{1}{\left(k^{2}\right)^{2}}\left(\ln \frac{k^{2}}{Q^{2}}\right)^{n} .
$$

Introducing

$$
\tilde{y}=\ln \frac{k^{2}}{Q^{2}}
$$


we arrive at

$$
D\left(Q^{2}\right)=\alpha_{s} \sum_{n=0}^{\infty}\left(\frac{\beta_{0} \alpha_{s}}{4 \pi}\right)^{n}(-1)^{n} \int d \tilde{y} \tilde{y}^{n} e^{-\tilde{y}}=\alpha_{s} \sum_{n=0}^{\infty}\left(\frac{\beta_{0} \alpha_{s}}{4 \pi}\right)^{n}(-1)^{n} n !
$$

This series is sign-alternating and, hence, is Borel-summable. The characteristic value of $\tilde{y}$ saturating the integral is $\tilde{y} \sim n$ implying $k^{2} \sim Q^{2} e^{n}$. Thus, at large $n$ we deal with large $k^{2}$ which explains why this contribution is referred to as the ultraviolet renormalon. It is well-defined per se. The best possible accuracy one can achieve with Eq. (20) compared to the exact result of the Borel transformation (3) is $\exp \left(-\frac{4 \pi}{\beta_{0} \alpha_{s}}\right) \sim \frac{\Lambda^{2}}{Q^{2}}$. I will not touch UV renormalons in what follows. Note that the singularities in the Borel-transform for the IR and UV renormalons have different separations from the origin 7 Namely,

$$
B_{D}\left(\alpha_{s}\right) \sim \begin{cases}\left(1-\frac{\beta_{0} \alpha_{s}}{8 \pi}\right)^{-1}, & \mathrm{IR} \\ \left(1+\frac{\beta_{0} \alpha_{s}}{4 \pi}\right)^{-1}, & \mathrm{UV} .\end{cases}
$$

The positions of the singularities in the $\alpha_{s}$ plane are

$$
\left(\alpha_{s}\right)_{*}=\left\{\begin{aligned}
\frac{8 \pi}{\beta_{0}}, & \mathrm{IR}, \\
-\frac{4 \pi}{\beta_{0}}, & \mathrm{UV} .
\end{aligned}\right.
$$

They are depicted in Fig. 3 along with the singularities due to instantons.

\section{Operator product expansion}

I remember that after the first seminar on the SVZ sum rules [43] in 1978 Eugene Bogomol'nyi used to ask me each time we met: "Look, how can you speak of power corrections in the two-point functions at large $Q^{2}$ when even

\footnotetext{
${ }^{7}$ As was mentioned above, Eq. (10) is simplified. Working with the exact formula [4] we would have obtained for the UV renormalon contribution in $B_{D}$ an expression that contains a single pole as well as a double pole at $\left(\alpha_{s}\right)_{*}=-\frac{4 \pi}{\beta_{0}}$. In addition to the term in the second line in (21) we would get another term $\sim\left(1+\frac{\beta_{0} \alpha_{s}}{4 \pi}\right)^{-2}$, see e.g. [11. No qualitative changes occur due to the presence of the double pole. I will not go into details, since the UV renormalons are mentioned only for completeness and will not be pursued further.
} 


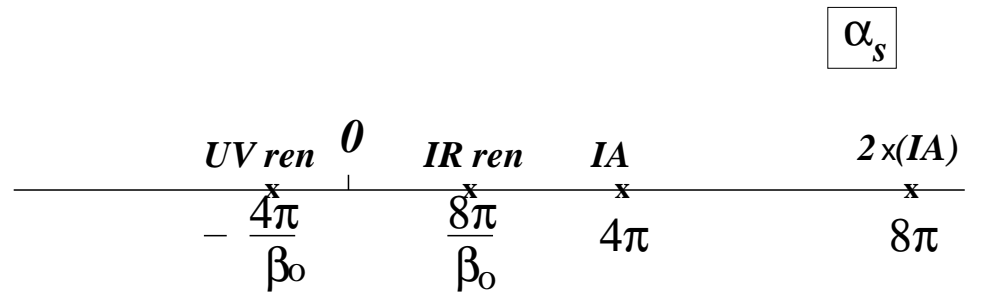

Figure 3: Singularities in the Borel plane.

the perturbative expansion (i.e. the expansion in $\left.1 / \ln \left(Q^{2} / \Lambda^{2}\right)\right)$ is not well defined? Isn't it an 'excessive force' abusive approach?"

At that time Wilson's wisdom only started to conquer the high-energy physics community. My understanding in 1978 missed some nuances too. In modern terminology what we mostly used in [43] is now known as the "practical" operator product expansion, its simplified version (see e.g. 14, Sect. 6, which is highly recommended to the reader unfamiliar with nuances of OPE in QCD). Although it is sufficient in solving problems arising in various applications and relatively easy to implement, in the present article in which I am after conceptual aspects, I have to be careful with formulations. Only then I will be able to answer the above Bogomoln'yi question in a positive way, namely:

"Consistent use of Wilson's OPE makes everything well-defined at the conceptual level. Technical implementation may not always be straightforward, however."

The operator product expansion (OPE) in asymptotically free theories is a book-keeping device separating short-distance (weak-coupling) contributions from those coming from large distances (strong coupling domain). To this end one introduces an auxiliary separation scale $\mu$. OPE is applicable whenever one deals with problems that can be formulated in the Euclidean space-time and in which one can regulate typical Euclidean distances by a varying large external momentum $Q$ (or $m_{Q}$ in the heavy quark problems). Wilson's OPE is meaningful if one can choose $\mu \ll Q$ (or $\mu \ll m_{Q}$ ), but $\mu \gg \Lambda$.

Note that a practical version that draws a divide in OPE between perturbation theory and nonperturbative effects is a simplification which may or may not be approximately valid, depending on the theory under consideration. The correct divide is between short- and large-distance contributions. As a book-keeping device of this type it cannot fail [13], provided no arith- 
metic mistake is made en route.

The idea of factorization of short and large distances, the central point of OPE, dates back to classical Wilson's work [44] (see also [45]) where it was put forward in connection with theories of strong interaction with conformal invariance at short distances. Shortly after, Wilson formulated a very general procedure of the renormalization-group flow (e.g. [45]) which became known as the Wilsonean renormalization group. Wilson's formulation makes no reference to perturbation theory, it applies both to strongly and weakly coupled theories. The focus of Wilson's work was on statistical physics, where the program is also known as the block-spin approach. Starting from the microscopic degrees of freedom at the shortest distances $a$, one "roughens" them, step by step, by constructing a sequence of effective (composite) degrees of freedom at distances $2 a, 4 a, 8 a$, and so on. At each given step $i$ one constructs an effective Hamiltonian, which fully accounts for dynamics at distances shorter than $a_{i}$ in the coefficient functions.

Surprisingly, in high-energy physics of the 1970s the framework of OPE was narrowed down to a very limited setting. On the theoretical side, it was discussed almost exclusively in perturbation theory, as is seen, for instance, from Refs. [46]. On the practical side, its applications were mostly narrowed down to deep inelastic scattering, where it was customary to work in the leading-twist approximation.

The general Wilson construction was adapted to QCD, for the systematic inclusion of power-suppressed effects, in [43, 13. Vacuum expectation value of the gluon density operator and other vacuum condensates were introduced for the first time, which allowed one to analyze a large number of vacuum two- and three-point functions, with quite nontrivial results. A consistent Wilsonean approach requires an auxiliary normalization point $\mu$ which plays the role of a "regulating" parameter separating hard contributions included in the coefficient functions and soft contributions residing in local operators occurring in the expansion. The degree of locality is regulated by the same parameter $\mu$.

Prevalent in the 1970s was a misconception that the OPE coefficients are determined exclusively by perturbation theory while the matrix elements of the operators involved are purely nonperturbative. Attempts to separate perturbation theory from "purely nonperturbative" condensates gave rise to inconsistencies (see e.g. [47]; I will return to this paper later) which questioned the very possibility of using the OPE-based methods in QCD.

In the heavy quark theory, in which Kolya's contribution was instrumen- 
tal, OPE acquired a new life constituting the basis of the heavy quark mass expansions (for a review see [10]). In this range of questions one deals with expectation values of various operators over the heavy quark meson or baryon states, rather than vacuum expectation values. The overall ideology does not change, however.

The OPE formalism provides a natural framework for the discussion of IR renormalons and how they should be treated in theories with strong coupling regime.

\section{An illustrative example}

Despite the conceptual simplicity of OPE, it continues to be questioned in the literature, in particular, in connection with renormalons in strongly coupled theories. The statement which I would like to illustrate in this section is: if one introduces the boundary point $\mu$ (unavoidable in non-conformal field theories) and abandons the idea of separation along the line "perturbative vs. nonperturbative," all would-be inconsistencies disappear, and so does the problem of renormalons.

Following [13] I will consider here a relatively simple example of a twodimensional model - the so-called $O(N)$ model - which has both, asymptotic freedom and renormalons, and at the same time is exactly solvable at large $N$. Classically excitations in this model are massless. A mass gap is generated at the quantum (nonperturbative) level. This example in the given context was suggested long ago in [48]. In this paper OPE (in its "practical" version) was found to be perfectly consistent with the exact solution in the leading in $1 / N$ approximation.

However, the subsequent exploration of composite operators [47] questioned the existence of consistently defined composite operators in OPE at the level of the first subleading correction (of the relative order of $1 / N$ ). Now, I will demonstrate how inconsistencies are eliminated once $\mu$ is explicitly introduced.

The Lagrangian of the model has the form [49] (for a review see [50])

$$
\mathcal{L}=\frac{N}{2 \lambda}\left(\partial_{\mu} S^{a}\right)\left(\partial^{\mu} S^{a}\right), \quad \vec{S}^{2}=1
$$

where $\vec{S}=\left\{S^{1}, S^{2}, \ldots, S^{N}\right\}$ is an $N$-component real (iso)vector field, and $\lambda$ is the 't Hooft coupling, which stays fixed in the limit $N \rightarrow \infty$. The $\mathrm{O}(N)$ 
symmetry of this Lagrangian is evident. This model is asymptotically free [50], in much the same way as Yang-Mills theory,

$$
\lambda(p)=\frac{2 \pi}{\ln \frac{p}{m}}, \quad \text { or } m=p \exp \left[-\frac{2 \pi}{\lambda(p)}\right],
$$

where $m$ is a dynamically generated mass gap. In perturbation theory the $\mathrm{O}(N)$ symmetry is spontaneously broken implying $N-1$ Goldstone modes. The $\mathrm{O}(N)$ symmetry is restored in the exact solution, in full accord with the Coleman theorem [51]. The vacuum condensates of the type $\left\langle\left(\partial_{\mu} S^{a}\right)\left(\partial^{\mu} S^{a}\right)\right\rangle \neq 0$ develop. To the leading order in $N$ (for details see e.g. [50])

$$
\left\langle\left[\left(\partial_{\mu} S^{a}\right)^{2}\right]^{k}\right\rangle=m^{2 k}, \quad k=1,2, \ldots
$$

In this order the above matrix elements scale as $N^{0}$ and factorize. To order $O\left(N^{0}\right)$ each of them is $\mu$ independent because in this order the anomalous dimension of the operator $\left(\partial_{\mu} S^{a}\right)^{2}$ vanishes. Needless to say, there are nonfactorizable corrections scaling as $1 / N$. For what follows it is convenient to introduce a special notation for the operator

$$
\left(\partial_{\mu} S^{a}\right)^{2} \equiv \alpha
$$

The operator basis in OPE to the order $O\left(N^{0}\right)$ consists of the composite operators of the type $\alpha^{n}$. In the subleading orders operators with an entangled index structure appear, but we do not have to consider them here.

To discuss OPE let us consider the two-point function

$$
\begin{aligned}
P\left(q^{2}\right) & =i \int d^{2} x e^{i q x}\left\langle T\left\{j_{s}(x) j_{s}(0)\right\}\right\rangle, \\
j_{s} & =\sqrt{N}\left(\partial_{\mu} S^{a}\right)^{2}
\end{aligned}
$$

at large (Euclidean) values of $q^{2}$ (i.e. $q^{2}$ negative and $Q^{2} \equiv-q^{2}$ positive).

The general OPE formula for the two-point function (27) (at large Euclidean $Q^{2}$ ) has the form

$$
P\left(Q^{2}\right)=c_{0}\left(Q^{2}, \mu^{2}\right) Q^{2} \mathbf{I}+c_{1}\left(Q^{2}, \mu^{2}\right) \alpha(\mu)+\frac{c_{2}\left(Q^{2}, \mu^{2}\right)}{Q^{2}}[\alpha(\mu)]^{2}+\ldots
$$

where $c_{i}$ are the coefficient functions. 
With our normalization $P\left(Q^{2}\right) \sim N^{0}$ in the leading order in $N$. All coefficient functions and expectations values scale in the same way, as $N^{0}$, with subleading $1 / N$ corrections. Moreover, to the leading order the OPE coefficients in (27) are $\mu$ independent, with no factorial divergences. As a result, at $N=\infty$ one can close one's eyes on subtleties and adhere to the simplified formula according to which the coefficient functions are determined exclusively by perturbation theory, and (large-distance) vacuum condensates exclusively by nonperturbative effects 8 This simplified formula is self-consistent [48].

An apparent inconsistency was noted at the level of $1 / N$ corrections [47]. Among many additional computations at this level one has to define composite operators beyond factorization, the simplest of which is the operator $\alpha^{2}$. Below I will show that introducing the normalization point $\mu$ - a necessary step not seen in [47] because of dimensional regularization in which the scale separation is not explicit - solves all would-be problems.

The vacuum expectation value of $\alpha^{2}$ can be defined as follows:

$$
\begin{aligned}
\left\langle[\alpha(\mu)]^{2}\right\rangle & =m^{4}+\left\langle[\alpha(\mu)]^{2}\right\rangle_{\mathrm{conn}}, \\
\left\langle[\alpha(\mu)]^{2}\right\rangle_{\mathrm{conn}} & =\int_{\operatorname{Eucl} p<\mu} \frac{d^{2} p}{(2 \pi)^{2}} D\left(p^{2}\right),
\end{aligned}
$$

where the subscript conn means the connected (nonfactorizable) part and $D\left(p^{2}\right)$ is the propagator of the $\alpha$ field known from the exact solution of the model to the leading order in $N$,

$$
D\left(p^{2}\right)=-\frac{4 \pi}{N} \frac{\sqrt{p^{2}\left(p^{2}+4 m^{2}\right)}}{\ln \frac{\sqrt{\left(p^{2}+4 m^{2}\right)}+\sqrt{p^{2}}}{\sqrt{\left(p^{2}+4 m^{2}\right)}-\sqrt{p^{2}}}},
$$

see Fig. 4 ,

It is obvious that the connected part is suppressed by $1 / N$ compared to the factorized part. For what follows it will be useful to rewrite the denominator in (30) at $p^{2} \gg m^{2}$ as

$$
\frac{1}{\ln \frac{\sqrt{\left(p^{2}+4 m^{2}\right)}+\sqrt{p^{2}}}{\sqrt{\left(p^{2}+4 m^{2}\right)}-\sqrt{p^{2}}}} \equiv \frac{\lambda(\mu)}{4 \pi}\left[1-\frac{\lambda(\mu)}{4 \pi} \ln \frac{\mu^{2}}{p^{2}}\right]^{-1}\left[1+O\left(\frac{m^{2}}{p^{2}}\right)\right] .
$$

\footnotetext{
${ }^{8}$ This exceptional situation specific to the $O(N)$ model has no parallel in QCD.
} 


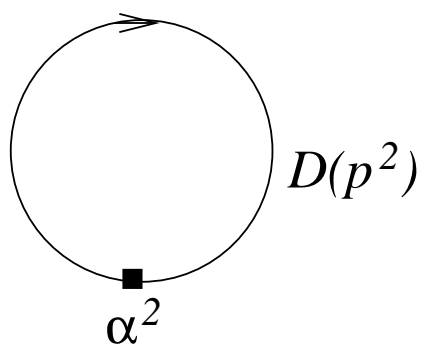

Figure 4: The leading contribution to $\left\langle[\alpha(\mu)]^{2}\right\rangle_{\text {conn }}$. The propagator of $\alpha$ is presented in (30).

This approximation certainly becomes meaningless at $p^{2} \leq m^{2}$ since the expression $1-\frac{\lambda(\mu)}{4 \pi} \ln \frac{\mu^{2}}{p^{2}}$ vanishes at $p^{2}=m^{2}$.

The integral in (29) is doable and can be expressed in terms of special functions [13]. We need to examine this integral in the limit $\mu \gg m$ because only in this limit the coefficient functions in OPE are predominantly perturbative. The result for $\left\langle[\alpha(\mu)]^{2}\right\rangle_{\text {conn }}$ includes terms $O\left(\mu^{4}\right), O\left(\mu^{2} m^{2}\right), O\left(m^{4}\right)$ and $O\left(m^{6} / \mu^{2}\right)$. For simplicity will omit the latter and focus on the first three terms. The second and the third can be established from the exact result (30); as we will see shortly, they do not contain factorial divergences at all. The first term does, see below. We could find it from the exact result too. But it will be more instructive to calculate it from the approximate formula (31). Then the relation to renormalons will become more apparent. Equation (31), being integrated from $m^{2}$ to $\mu^{2}$ (taking account of the remark after (31)), is perfectly sufficient to determine the $\mu^{4}$ term in $\left\langle[\alpha(\mu)]^{2}\right\rangle_{\text {conn. Indeed, }}$ substituting (31) in (30) and keeping $m$ only in the argument of logarithms we obtain

$$
\begin{aligned}
\left\langle[\alpha(\mu)]^{2}\right\rangle_{\text {conn }} & =-\frac{1}{N} \int_{m^{2}}^{\mu^{2}} p^{2} d p^{2} \frac{\lambda(\mu)}{4 \pi} \sum_{k=0}^{\infty}\left(\frac{\lambda(\mu)}{4 \pi} \ln \frac{\mu^{2}}{p^{2}}\right)^{k} \\
& =-\frac{1}{N} \mu^{4} \sum_{k=0}^{\infty}\left(\frac{\lambda(\mu)}{8 \pi}\right)^{k+1} \int_{0}^{k_{*}} d y y^{k} e^{-y}
\end{aligned}
$$

where

$$
k_{*}=2 \ln \frac{\mu^{2}}{m^{2}} .
$$

If $k \leq k_{*}$ the integral in the right-hand side of (32) can be extended to infinity 
since the saddle point lies at $y=k$, and then this integral produces $k$ !,

$$
\left\langle[\alpha(\mu)]^{2}\right\rangle_{\mathrm{conn}}=-\frac{1}{N} \mu^{4} \sum_{k=0}^{k_{*}}\left(\frac{\lambda(\mu)}{8 \pi}\right)^{k+1} k ! .
$$

If $k>k_{*}$ the integral is saturated at $y=k_{*}$, the factorial growth ceases to continue and the right-hand side of (32) reduces to $O\left(m^{4}\right)$.

The exact expression for $\left\langle[\alpha(\mu)]^{2}\right\rangle_{\text {conn }}$ is well-defined and can be explicitly calculated. I omit a number of simple algebraic manipulations referring the reader to the original paper [13]. The result is: 9 ]

$$
\begin{aligned}
\left\langle[\alpha(\mu)]^{2}\right\rangle_{\text {conn }} & =\frac{1}{N}\left\{-\mu^{4}\left[e^{-L} \operatorname{Ei}(L)\right]\right. \\
& \left.-\frac{4}{L} \mu^{2} m^{2}+2 m^{4}\left(C+\ln L-\frac{1}{L}+\frac{5}{L^{2}}\right)\right\},
\end{aligned}
$$

where

$$
L=2 \ln \frac{\mu^{2}}{m^{2}}=\left[\frac{\lambda(\mu)}{8 \pi}\right]^{-1},
$$

and $C \approx 0.5772 \ldots$ is the Euler constant. The $O\left(\mu^{4}\right)$ terms in (34) and (35) perfectly match each other!

Needless to say, $P\left(Q^{2}\right)$ does not contain the auxiliary parameter $\mu$; it depends only on physical parameters $Q^{2}$ and $m^{2}$. This means that in the right-hand side of (28) $\mu$ must cancel. As was mentioned, at order $N^{0}$ is does not appear at all. The first and the second term in (35) appearing at the level $O(1 / N)$ must be canceled by the corresponding contributions coming from the first and the second term in (28)). And they do, indeed! The coefficient $c_{0}$ has a correction $\frac{\mu^{4}}{Q^{4}} \frac{1}{N}\left[e^{-L} \operatorname{Ei}(L)\right]$ while $c_{1}$ has $\lambda(\mu) \frac{\mu^{2}}{Q^{2}}$.

The terms proportional to $m^{4}$ in Eq. (35) do not cancel. They still have a weak (logarithmic) dependence on $\mu$ through $L^{-1}$ and $\ln L$. This is a manifestation of the anomalous dimension of the operator $[\alpha(\mu)]^{2}$ which shows up beyond the leading (factorization) order. It is canceled by the corresponding logarithmic terms in $c_{2}\left(Q^{2}, \mu^{2}\right)$.

The reader interested in additional details is referred to [52] for a later discussion of OPE in a particular correlation function in the $\mathrm{O}(N)$ model at

\footnotetext{
${ }^{9} \mathrm{I}$ correct here a number of misprints in the expression for $\left\langle[\alpha(\mu)]^{2}\right\rangle_{\text {conn }}$ given in [13].
} 
the subleasing level (i.e. $\mathrm{O}(1 / N)$ corrections) 10

Concluding this section let me mention that the $\mathrm{O}(N)$ sigma model is promising in one more aspect: In this model at $N>3$ instantons disappear, while nothing dramatic happens to renormalons. Question: what replaces the instanton singularities in the Borel plane?

\section{$9 \quad \mathrm{OPE}$ and renormalons in QCD}

After this brief digression intended to demonstrate peculiarities of perturbation theory in strongly coupled models with the known solution let us return to QCD where no exact solution is available. I will start from correlation functions of the type (7) at large Euclidean $q^{2}$ in which OPE can be consistently built through separation of large- and short-distance contributions. For simplicity, for our illustrative purposes, I will set the separation scale at $\mu=\Lambda$ rather than at $\mu \gg \Lambda$. This would be inappropriate in quantitative analyses; however, my task is to reveal qualitative aspects. For this purpose no harm will be done if I put $\mu=\Lambda$. With this convention all relevant expressions will dramatically simplify.

Let us have a closer look at Eqs. (10) and (11). The unlimited factorial divergence in (16) is a direct consequence of integration over $k^{2}$ in (12) all the way down to $k^{2}=0$. Not only this is nonsensical because of the pole in (11) at $k^{2}=\Lambda^{2}$, this is not what we should do in calculating coefficient functions in OPE. The coefficients must include $k^{2}>\Lambda^{2}$ by construction. The domain of small $k^{2}$ (below $\Lambda^{2}$ ) must be excluded from $c_{0}$ and referred to the vacuum matrix element of the gluon operator $G_{\mu \nu}^{2}$. Indeed, in the sum in Eq. (12) all terms with $n>n_{*}$ can be written as (see Fig. 2)

$$
\begin{aligned}
\Delta D\left(Q^{2}\right) & =\frac{\alpha_{s}}{2} \sum_{n>n_{*}}\left(\frac{\beta_{0} \alpha_{s}}{8 \pi}\right)^{n} n_{*}^{n} e^{-n_{*}} \\
& =\frac{\alpha_{s}}{2} \sum_{n>n_{*}} \frac{\Lambda^{4}}{Q^{4}}
\end{aligned}
$$

where I used the fact that $\frac{\beta_{0} \alpha_{s}\left(Q^{2}\right)}{8 \pi}=\frac{1}{2 \ln \left(Q^{2} / \Lambda^{2}\right)}=1 / n_{*}$. Of course, we

\footnotetext{
${ }^{10} \mathrm{~A}$ remarkable feature making the model different from QCD is the fact of OPE convergence at the level of $\mathrm{O}\left(N^{0}\right)$ and $\mathrm{O}\left(N^{-1}\right)$ terms. This is due the fact that at this level particle production thresholds do not extend to infinite energies in the $\mathrm{O}(N)$ sigma model, unlike QCD.
} 
cannot calculate the gluon condensate from the above expression for the tail of the series (12) representing the large distance contribution, for a number of reasons. In particular, the value of the coefficient in front of $\Lambda^{4} / Q^{4}$ remains uncertain in (37) because Eq. (11) is no longer valid at such momenta. We do not expect the gluon Green functions used in calculation in Fig. 1 and in Eq. (11) to retain any meaning in the nonperturbative domain of strong coupling dynamics. A qualitative feature - the power dependence $(\Lambda / Q)^{4}$ in (37) - is correct, however.

We note with satisfaction that the fourth power of the parameter $\Lambda / Q$ which we find from this tail exactly matches the OPE contribution of the operator $\left\langle G_{\mu \nu}^{2}\right\rangle$. In Sect. 8 where we analyzed an exactly solvable model we could convince ourselves that this is not a coincidence.

Summarizing this section I can say that consistent use of OPE cures the problem of the renormalon-related factorial divergence of the coefficients in the $\alpha_{s}$ series, absorbing the IR tail of the series in the vacuum expectation value of the gluon operator $G_{\mu \nu}^{2}$ and similar higher-order operators. Although the value of $\left\langle G_{\mu \nu}^{2}\right\rangle$ cannot be calculated from renormalons, the very fact of its existence can be established.

\section{Sources of factorials and master formula}

From quantum mechanics we learn that the factorial divergence can arise from "soft" fields, e.g. instantons (see Sect. 5). In QCD the instantons are ill-defined in the IR and, strictly speaking, nobody knows what to do with them 11 There is a perfectly legitimate conceptual way out, however. If one considers QCD in the 't Hooft limit of large number of colors [38, instantons decouple. At the same time, none of the essential features of QCD disappears. In addition to phenomenological arguments [54], this statement is supported by an exact solution of a strongly coupled two-dimensional model with asymptotic freedom [55].

$* * * * *$

Now I will try to summarize the lessons we learned in a single (simplified) "master" formula. At large Euclidean momenta the correlation functions of

\footnotetext{
${ }^{11}$ This statement is an exaggeration. The inquisitive reader is referred to [53] for an alternative point of view on instantons in QCD vacuum.
} 
the type (7) and similar can be represented as

$$
\begin{aligned}
D\left(Q^{2}\right) & =\sum_{n=0}^{n_{*}^{0}} c_{0, n}\left(\frac{1}{\ln Q^{2} / \Lambda^{2}}\right)^{n} \\
& +\sum_{n=0}^{n_{*}^{1}} c_{1, n}\left(\frac{1}{\ln Q^{2} / \Lambda^{2}}\right)^{n}\left(\frac{\Lambda}{Q}\right)^{d_{1}} \\
& +\sum_{n=0}^{n_{*}^{2}} c_{2, n}\left(\frac{1}{\ln Q^{2} / \Lambda^{2}}\right)^{n}\left(\frac{\Lambda}{Q}\right)^{d_{2}}+\ldots \\
& + \text { "exponential terms". }
\end{aligned}
$$

Equation (38) is simplified in a number of ways. First, it is assumed that the currents in the left-hand side have no anomalous dimensions, and so do the operators appearing on the right-hand side. They are assumed to have only normal dimensions given by $d_{i}$ for the $i$-th operator. Second, I ignore the second and all higher coefficients in the $\beta$ function so that the running coupling is represented by a pure logarithm. All these assumptions are not realistic in QCD 12 I stick to them to make the master formula concise. Inclusion of higher orders in the $\beta$ function and anomalous dimensions both on the left- and right-hand sides will give rise to rather contrived additional terms and factors containing $\log \log$ 's, $\log \log \log$ 's $(\log \log / \log )$ 's, etc. This is a purely technical, rather than conceptual, complication, however.

So far I discussed the convergence of the perturbative series (explaining that the regulating parameter $\mu$ in OPE allows one to make them meaningful). The expansion (38) runs not only in powers of $1 / \ln Q^{2}$, but also in powers of $\Lambda / Q$. This is a double expansion, and the power series in $\Lambda / Q$ is also infinite in its turn. Does it have a finite radius of convergence?

Needless to say, this is an important question. The answer to it is negative 13 As was argued in [56, 14, power series are factorially divergent in high orders. This is a rather straightforward observation following from the analytic structure of $D\left(Q^{2}\right)$. In a nut shell, since the cut in $D\left(Q^{2}\right)$ runs all the way to infinity along the positive real semi-axis of $q^{2}$, the $1 / Q^{2}$ expansion cannot be convergent. The last line in Eq. (38) symbolically represents a divergent tail of the power series.

\footnotetext{
${ }^{12}$ They could be made somewhat more realistic in $\mathcal{N}=2$ super-Yang-Mills.

${ }^{13}$ See also foot note 10.
} 
The actual argument is somewhat more subtle than that, but the final conclusion - that high-order tail of the (divergent) power series gives rise to exponentially small corrections (exponentially small in Euclidean, oscillating in Minkowski) - still holds. The most instructive way to see it is provided by a toy model presented in Sect. 2.2 of [14] which refers to the 't Hooft limit. Then qualitatively one can saturate $\Pi\left(Q^{2}\right)$ by an infinite comb of equidistant infinitely narrow resonances. For simplicity one can assume that the couplings of these resonances to the current do not depend on the excitation number. Then 14

$$
\Pi\left(Q^{2}\right)=-\frac{N_{c}}{12 \pi^{2}} \psi(z)+\text { const }
$$

where

$$
z=\frac{Q^{2}+m_{\rho}^{2}}{3 m_{\rho}^{2}},
$$

and $\psi(z)$ is Euler's $\psi$ function. In the Euclidean domain of positive $Q^{2}$

$$
\ln z \sim-\frac{1}{2 z}-\sum_{n=1}^{\infty} \frac{B_{2 n}}{2 n} \frac{1}{z^{2 n}},
$$

$B_{2 n}$ stand for the Bernoulli numbers

$$
B_{2 n}=(-1)^{n} \frac{2(2 n) !}{(2 \pi)^{2 n}} \zeta(2 n)
$$

and $\zeta$ is the Riemann function. The tilde " $\sim$ " in (41) means that the series in $1 / Q^{2}$ is asymptotic: since $\zeta(2 n) \sim 1$ the expansion coefficients in (41) are obviously factorially divergent. The tail of the $1 / Q^{2}$ series after optimal truncation is exponentially small. Alternatively, one can apply the Borel procedure since the alternating signs in (42) indicate Borel summability,

$$
\left(\frac{1}{Q^{2}}\right)^{n} \rightarrow \frac{1}{(n-1) !}\left(\frac{1}{M^{2}}\right)^{n}
$$

The position of the singularity in the $1 / M^{2}$ plane is $2 /\left(3 m_{\rho}^{2}\right)$.

\footnotetext{
${ }^{14}$ The factor 3 in the denominator of (40) is an approximate empiric number.
} 


\section{A breakthrough idea}

Now I am finally ready to explain the idea first put forward in [15, 16]. As was elucidated above, in the processes with OPE renormalons play no special role as long as the operator basis in OPE is complete, no relevant operator is accidentally omitted. However, there exists a wide range of phenomena at high energies (or in heavy quark physics) which do not allow one to carry out OPE-based analyses. The most well-known example of this type is jet physics. Up to a certain time these processes were treated exclusively in the realm of perturbative QCD. An estimate of nonperturbative effects, even as approximate as it could be, was badly needed. A minimalistic and urgent task was to find the power of $1 / E$ (or $1 / m_{Q}$ ) which controls the degree of fall-off of the leading nonperturbative effect.

To this end it was suggested [15, 16] to analyze the tails of the renormalon series. I hasten to add that renormalons by no means capture all nonperturbative effects. For instance, they are blind to any effects due to chiral symmetry breaking. Thus, they cannot guide us if chiral symmetry breaking plays a role. Hints associated with renormalons refer to gluons.

The first example of the "renormalon guidance" (that later proliferated to many other analyses) was the so-called heavy quark pole mass. The heavy quark mass is a key parameter in most aspects of heavy quark physics. The pole mass was routinely used in analyzing data. It is well-defined (infrared stable) and unambiguous to any finite order in perturbation theory. This infrared stability could give an impression that the pole mass is well-defined in general. This misinterpretation was quite common in the literature in the early 1990s.

The fact that the pole mass is not well-defined at the nonperturbative level was first noted and emphasized in [15, 16]. What is even more important, a rather powerful renormalon-based tool was suggested for evaluating the corresponding nonperturbative contribution. The problem arises because the pole mass is sensitive to large distance dynamics, although this fact is not obvious in perturbative calculations. Infrared contributions lead to an intrinsic uncertainty in the pole mass of order $\Lambda$, i.e. a $\Lambda / m_{Q}$ power correction. Renormalons produce clear evidence for this non-perturbative correction to $m_{Q}^{\text {pole }}$. The signal comes from the factorial growth of the high order terms in the $\alpha_{s}$ expansion corresponding to a singularity residing at $2 \pi / \beta_{0}$ in the Borel plane.

The renormalon contribution to the pole mass is shown in Fig. 3. The 


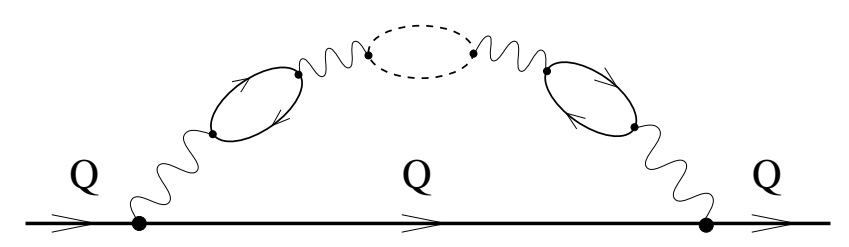

Figure 5: Perturbative diagrams leading to the IR renormalon uncertainty in $m_{Q}^{\text {pole }}$ of order $\Lambda$. The number of bubble insertions in the gluon propagator can be arbitrary.

bubble chain generates the running of the strong coupling $\alpha_{s}$. To leading order, it can be accounted for by inserting the running coupling constant $\alpha_{s}\left(k^{2}\right)$ in the integrand corresponding to the one-loop expression. In the nonrelativistic regime, when the internal momentum $|k| \ll m_{Q}$, the expression is simple,

$$
\delta m_{Q} \sim-\frac{4}{3} \int \frac{d^{4} k}{(2 \pi)^{4} i k_{0}} \frac{4 \pi \alpha_{s}\left(-k^{2}\right)}{k^{2}}=\frac{4}{3} \int \frac{d^{3} \vec{k}}{4 \pi^{2}} \frac{\alpha_{s}\left(\vec{k}^{2}\right)}{\vec{k}^{2}},
$$

where $\alpha_{s}\left(\vec{k}^{2}\right)$ can be read off from Eq. (11) with the substitution $Q^{2} \rightarrow m_{Q}^{2}$. Expressing the running $\alpha_{s}\left(k^{2}\right)$ in terms of $\alpha_{s}\left(m_{Q}^{2}\right)$ (note that $\vec{k}^{2}<m_{Q}^{2}$ ), and expanding in $\alpha_{s}\left(m_{Q}^{2}\right)$ we arrive at

$$
\frac{\delta m_{Q}^{(n+1)}}{m_{Q}} \sim \frac{4}{3} \frac{\alpha_{s}\left(m_{Q}^{2}\right)}{\pi} n !\left(\frac{\beta_{0} \alpha_{s}\left(m_{Q}^{2}\right)}{2 \pi}\right)^{n} .
$$

The right-hand side represents the renormalon series. This series is factorially divergent and is not Borel-summable. Moreover, in the case at hand there is no OPE which could absorb this tail in a higher-dimension operator. What should we do?

The question was posed and the answer given in [15, 16]: Following the line of reasoning applied in OPE-based processes we should truncate the series at an optimal order and, in addition, introduce an infrared parameter $\delta m_{Q}$ which will absorb the renormalon tail. Equation (45) implies that

$$
n_{*} \sim \ln \frac{m_{Q}}{\Lambda}, \quad \frac{\delta m_{Q}}{m_{Q}} \sim e^{-n_{*}} \sim \frac{\Lambda}{m_{Q}} .
$$

The perturbative expansion per se anticipates the onset of the nonperturbative regime (the impossibility of pinning down the would-be quark pole in perturbation theory to accuracy better than $\Lambda$ ). 
Certainly, the renormalons do not represent the dominant component of the infrared dynamics. However, they provide the "renormalon guidance" playing a very important role of an indicator of the presence of the powersuppressed nonperturbative effects.

\section{Renormalons in weak coupling problems}

In Sect. 4 it was mentioned that the study of renormalons at weak coupling can can shed new light on the general structure of field theory. According to the conjecture formulated in [34, 35] at weak coupling any particular factorially divergent contribution, if Borel-nonsummable, must match a certain quasiclassical field configuration. Such configurations were identified [35] in two-dimensional $C P(N-1)$ models which present a close parallel [50] to four-dimensional Yang-Mills. However, subtle details are not yet satisfactory.

The instanton quarks supposedly matching the perturbative factorial divergence in cylindrical geometry have action $4 \pi /\left(N g^{2}\right)$ resulting in singularities at $4 \pi k / N$ in the Borel plane 15 here $k=1,2, \ldots, N$ is an integer. If we have a look at Fig. 3, we will see that the positions of the renormalon singularities are quantized in the units of $4 \pi / \beta_{0}$. A matching relationship can only be achieved if $\beta_{0}=$ integer $\times N$. This is the case in $C P(N-1)$ models, but this is certainly not the case in Yang-Mills 16

Let us discuss this example - the two-dimensional $C P(N-1)$ model - in more detail. Assume it is considered on a cylinder $R_{1} \times S_{1}(r)$ where $r$ is the radius of the circle. At $r \rightarrow 0$ the problem reduces to quantummechanical, with the perturbative expansion being nonsummable à la Borel. The factorial divergence is not directly related to renormalons (which are absent in quantum mechanics), but it exists.

The corresponding singularity in the $g^{2}$ Borel plane lies at $8 \pi / N$. This happens to be exactly the action of two instanton quarks [35]. Thus, one

\footnotetext{
${ }^{15}$ In four dimensions it is convenient to consider the $\alpha$ plane, with $\alpha \equiv g^{2} / 4 \pi$. In two dimensions the $g^{2}$ plane is more convenient.

${ }^{16}$ There is an observation which, perhaps, gives hope for the future. In pure Yang-Mills $\beta_{0}=\frac{11}{3} N$. As was noted by Khriplovich long ago [57] (see also [58, Sect. 25.1) the above value of $\beta_{0}$ has a distinct two-component structure. If one calculates $\beta_{0}$ in the physical gauge without ghosts (e.g. Coulomb), one will discover that in fact $\beta_{0}=4 N-\frac{1}{3} N$ where the first term in the right-hand side presents antiscreening inherent only to non-Abelian gauge theories, while the second term, with the fractional coefficient, is a conventional screening.
} 
can (and does) achieve resurgence through construction of the corresponding trans-series. What is important for the following paragraph is the fact that action of two instanton quarks corresponds to the dimension of the lowest nontrivial operator in OPE, namely $(\partial S)^{2}$.

If $r \gtrsim \Lambda^{-1}$, we find ourselves in the strong coupling regime, the parameter $r^{-1}$ plays the role of $\mu$, and the perturbative factorial divergence is generated by renormalons, which should be treated, as usual, in the framework of OPE. Remarkably, the position of singularity in the Borel plane does not shift from $8 \pi / N$. OPE explains the renormalon singularity at $8 \pi / N$ since the first operator in OPE (after the trivial operator) has exactly the needed dimension and generates terms $\exp \left(-\frac{8 \pi}{N}\right) \sim \Lambda^{2}$. Thus, it is not ruled out that the positions of the leading singularity in the Borel plane is $r$-independent in the interval $r \in\left(0\right.$, const $\left.\times \Lambda^{-1}\right)$.

This $r$ independence cannot survive in Yang-Mills theories, because of the $\beta_{0}$ factor mentioned above. What can happen in Yang-Mills, however, with luck, is a smooth $r$-dependence of the singularity positions in the Borel plane. This question remains open.

Cylindrical geometry exploited in [34, 35] is not the only way to make Yang-Mills theory weakly coupled. Alternatively, one can Higgs the theory.

Assume we have SU(2) Yang-Mills theory fully Higgsed by an expectation value of the Higgs doublet field, just as in the standard model. The theory is at weak coupling. Assume we introduce $2 n_{f}$ doublets of chiral (Weyl) fermions $\left(\chi_{\alpha}^{i}\right)^{j}$ and $\left(\psi_{\alpha}^{i}\right)^{j}$, where $\alpha$ and $i$ are the Lorentz and SU(2) gauge indices, respectively, and $j=1,2, \ldots, n_{f}$. For simplicity we will assume that both the fermion and the Higgs masses are the same as the mass of the $W$ bosons $M$. After Higgsing this theory still has a global $\mathrm{SU}(2)$ symmetry. Three $W$ bosons form a triplet under this global SU(2).

This $\mathrm{SU}(2)$ theory has no internal anomalies. In fact, it is vector-like. With the even number of doublets it avoids Witten's global anomaly too [59, 58].

In this fully IR regularized theory we can repeat the analysis outlined in Sect. 6. The renormalon diagram in Fig. 11 now yields an expression similar to that in (12) at $k^{2} \gg M^{2}$. However, at $k^{2} \lesssim M^{2}$ the integral over $k^{2}$ in (12) must be cut off from below at $M^{2}$ (with logarithmic accuracy).

Now, in perturbation theory the large- $Q^{2}$ expansion of the Adler function 
has the form

$$
\begin{aligned}
D\left(Q^{2}\right) & =\sum c_{0, n}\left(\frac{1}{\ln Q^{2} / \Lambda^{2}}\right)^{n}+\sum c_{1, n}\left(\frac{1}{\ln Q^{2} / \Lambda^{2}}\right)^{n}\left(\frac{M}{Q}\right)^{2} \\
& +\sum c_{2, n}\left(\frac{1}{\ln Q^{2} / \Lambda^{2}}\right)^{n}\left(\frac{M}{Q}\right)^{4}+\ldots
\end{aligned}
$$

It is not difficult to see that (although the critical value of $n$ for the diagram in Fig. 1] changes compared to that in Sect. 6) the renormalon tail gives rise

to a residual term in the second line proportional to $(\Lambda / Q)^{4}$, i.e. similar to what we have in the limit $M=0$.

The weak coupling conjecture [34, 35] assumes that (47) (more exactly, the $(\Lambda / Q)^{4}$ term representing its tail) must match a certain quasiclassical field configuration. At the moment the only candidate I see is the instantonantiinstanton pair at a fixed (and small) separation. Is this the case? Can such a match be explicitly traced?

\section{Conclusions}

1) Twenty years after its emergence [15, 16], the renormalon counting remains the only known method for evaluating nonperturbative corrections in the processes without OPE.

2) Operator product expansion, with an explicit separation scale $\mu$, conceptually solves the problem of factorial divergence of the perturbative series, at least at $N \rightarrow \infty$;

3) Factorial divergence of the $(\Lambda / Q)^{k}$ series emerging in OPE at large $k$, as established in [56], needs further explorations and an appropriate theoretical description/understanding.

4) The resurgence program put forward in [34, 35] outlines a clear-cut parallel between factorial divergences at weak coupling on the one hand, which, being treated à la Ecalle, result in well-defined trans-series, and the OPE-based paradigm at strong coupling, on the other hand. More thinking is required to completely understand their relationship.

5) There are theories with renormalon-induced factorial divergence but no instantons (e.g. two-dimensional $\mathrm{O}(N)$ sigma model with $N>3$ ). Construction of OPE in such models is not affected by the absence of instantons. If 
one considers them in cylindrical geometry, at weak coupling, finding substitutes for instanton quarks is a challenge. The first steps in this direction have been made, but more work is needed.

\section{Acknowledgments}

I am grateful to Martin Beneke, Ikaros Bigi, Alexei Cherman, Gerald Dunne, Sergei Monin, Mithat Ünsal, and Arkady Vainshtein for useful comments.

This work is supported in part by DOE grant DE-FG02-94ER-40823.

\section{Appendix: Definitions}

We use the following convention for the $\beta$ function:

$$
\beta\left(\alpha_{s}\right)=\mu \frac{\partial \alpha_{s}}{\partial \mu}=-\frac{1}{2 \pi} \beta_{0} \alpha_{s}^{2}+\frac{1}{2(2 \pi)^{2}} \beta_{1} \alpha_{s}^{3}+\ldots
$$

where 60]

$$
\beta_{0} \equiv \beta_{\text {ogluon }}+\beta_{0}^{f}=\frac{11}{3} N_{c}-\frac{2}{3} N_{f}
$$

and

$$
\beta_{1}=-2\left[\frac{17}{3} N_{c}^{2}-\frac{N_{f}}{6 N_{c}}\left(13 N_{c}^{2}-3\right)\right] .
$$

For three massless flavors $\beta_{0}=9$. The first two coefficients of the $\beta$ function are scheme-independent.

\section{References}

[1] V. A. Khoze, M. A. Shifman, N. G. Uraltsev and M. B. Voloshin, Sov. J. Nucl. Phys. 46, 112 (1987).

[2] I. I. Y. Bigi, V. A. Khoze, N. G. Uraltsev and A. I. Sanda, The Question of CP Noninvariance - as Seen Through the Eyes of Neutral Beauty, Adv. Ser. Direct. High Energy Phys. 3, 175 (1989).

[3] I. Bigi, this Volume. 
[4] M. A. Shifman, Int. J. Mod. Phys. A 14, 5017 (1999) hep-th/9906049].

[5] M. A. Shifman and M. B. Voloshin, Sov. J. Nucl. Phys. 41, 120 (1985). Sov. Phys. JETP 64, 698 (1986). Sov. J. Nucl. Phys. 45, 292 (1987).

[6] H. Georgi, Phys. Lett. B 240, 447 (1990).

[7] N. Isgur and M. B. Wise, Phys. Lett. B 232, 113 (1989).

[8] I. I. Y. Bigi, N. G. Uraltsev and A. I. Vainshtein, Phys. Lett. B 293, 430 (1992) [Erratum-ibid. B 297, 477 (1993)] [hep-ph/9207214].

[9] J. Chay, H. Georgi and B. Grinstein, Phys. Lett. B 247, 399 (1990).

[10] M. A. Shifman, Lectures on heavy quarks in quantum chromodynamics, in M. Shifman, ITEP Lectures on Particle Physics and Field Theory, (World Scientific, Sigapore, 1999), Vol. 1, p. 1 [hep-ph/9510377].

[11] M. Beneke, Phys. Rept. 317, 1 (1999) [hep-ph/9807443]; M. Beneke and V. M. Braun, Renormalons and power corrections, in At the Frontier of Particle Physics, Ed. M. Shifman (World Scientific, Singapore, 2001), Vol. 3, p. 1719 [hep-ph/0010208].

[12] A. Manohar and M. Wise, Heavy Quark Physics, (Cambridge University Press, 2000).

[13] V. A. Novikov, M. A. Shifman, A. I. Vainshtein and V. I. Zakharov, Nucl. Phys. B 249, 445 (1985).

[14] M. A. Shifman, Prog. Theor. Phys. Suppl. 131, 1 (1998) hep-ph/9802214.

[15] I. I. Y. Bigi, M. A. Shifman, N. G. Uraltsev and A. I. Vainshtein, Phys. Rev. D 50, 2234 (1994) hep-ph/9402360].

[16] M. Beneke and V. M. Braun, Nucl. Phys. B 426, 301 (1994) hep-ph/9402364.

[17] B. R. Webber, Phys. Lett. B 339, 148 (1994) hep-ph/9408222.

[18] F. J. Dyson, Phys. Rev. 85, 631 (1952). 
[19] Large-Order Behavior of Perturbation Theory, ed. J.C. Le Guillou and J. Zinn-Justin (North-Holland, Amsterdam, 1990).

[20] W. E. Thirring, Helv. Phys. Acta 26, 33 (1953).

[21] L. N. Lipatov, Sov. Phys. JETP 45, 216 (1977).

[22] E. B. Bogomolny and V. A. Fateev, Phys. Lett. B 76, 210 (1978).

[23] A. I. Vainshtein, Decaying Systems and Divergence of the Series of Perturbation Theory, Novosibirsk preprint, 1964. Its English translation was first published in [24].

[24] A.I. Vainshtein, in Continuous Advances in QCD 2002, ArkadyFest, ed. K. Olive, M. Shifman, and M. Voloshin (World Scientific, Singapore, 2002), p. 619 .

[25] C. M. Bender and T. T. Wu, Phys. Rev. D 7, 1620 (1973).

[26] G. 't Hooft, Can We Make Sense Out of Quantum Chromodynamics? in The Whys Of Subnuclear Physics, Erice 1977 ed. A. Zichichi (Plenum, New York, 1979), p. 943; B. E. Lautrup, Phys. Lett. B 69, 109 (1977); G. Parisi, Phys. Lett. B 76, 65 (1978); Nucl. Phys. B 150, 163 (1979); A. H. Mueller, Nucl. Phys. B 250, 327 (1985).

[27] J. Zinn-Justin, Phys. Rept. 70, 109 (1981); Quantum Field Theory and Critical Phenomena (Clarendon Press, Oxford, 1999).

[28] E. B. Bogomolny, Phys. Lett. B 91, 431 (1980).

[29] J. Zinn-Justin, Nucl. Phys. B 192, 125 (1981).

[30] G. V. Dunne and M. Ünsal, JHEP 1211, 170 (2012) arXiv:1210.2423 [hep-th]].

[31] J. Zinn-Justin and U. D. Jentschura, Phys. Lett. B 596, 138 (2004) hep-ph/0405279]; Annals Phys. 313, 197 (2004) quant-ph/0501136; Annals Phys. 313, 269 (2004) quant-ph/0501137.

[32] G. V. Dunne and M. Ünsal, Generating Energy Eigenvalue Trans-series from Perturbation Theory, arXiv:1306.4405 [hep-th]. 
[33] I. Aniceto and R. Schiappa, Nonperturbative Ambiguities and the Reality of Resurgent Trans-series, arXiv:1308.1115 [hep-th].

[34] P. Argyres and M. Ünsal, Phys. Rev. Lett. 109, 121601 (2012) arXiv:1204.1661 [hep-th]]; JHEP 1208, 063 (2012) arXiv:1206.1890 [hep-th]].

[35] G. V. Dunne and M. Ünsal, Phys. Rev. D 87, 025015 (2013) arXiv:1210.3646 [hep-th]].

[36] M. Ünsal, Phys. Rev. Lett. 100, 032005 (2008) arXiv:0708.1772 [hepth]].

[37] M. Ünsal, Phys. Rev. D 80, 065001 (2009) arXiv:0709.3269 [hep-th]].

[38] G. 't Hooft, Nucl. Phys. B 72, 461 (1974); see also Planar Diagram Field Theories, in G. 't Hooft, Under the Spell of the Gauge Principle, World Scientific, Singapore, 1994), p. 378; Commun. Math. Phys. 86, 449 (1982).

[39] J. Koplik, A. Neveu and S. Nussinov, Nucl. Phys. B 123, 109 (1977).

[40] A. H. Mueller, in Proc. Int. Conf. QCD - 20 Years Later, Aachen 1992, eds. P. Zerwas and H. Kastrup, (World Scientific, Singapore, 1993), vol. 1 , page 162 .

[41] M. Neubert, Phys. Rev. D 51, 5924 (1995) [hep-ph/9412265].

[42] V. I. Zakharov, Nucl. Phys. B 385, 452 (1992).

[43] M. A. Shifman, A. I. Vainshtein and V. I. Zakharov, Nucl. Phys. B 147, 385 (1979).

[44] K. G. Wilson, Phys. Rev. 179, 1499 (1969).

[45] K. G. Wilson and J. B. Kogut, Phys. Rept. 12, 75 (1974).

[46] K. Symanzik, Commun. Math. Phys. 23, 49 (1971); C. G. Callan, Jr., Phys. Rev. D 5, 3202 (1972); W. Zimmermann, in Lectures on Elementary Particles and Quantum Field Theory, Eds. S. Deser, M. Grisaru, and H. Pendelton (MIT Press, Cambridge, MA., 1971), Vol. 1. 
[47] F. David, Nucl. Phys. B 234, 237 (1984).

[48] F. David, Nucl. Phys. B 209, 433 (1982).

[49] W. A. Bardeen, B. W. Lee and R. E. Shrock, Phys. Rev. D 14, 985 (1976).

[50] V. A. Novikov, M. A. Shifman, A. I. Vainshtein and V. I. Zakharov, Phys. Rept. 116, 103 (1984).

[51] S. R. Coleman, Commun. Math. Phys. 31, 259 (1973).

[52] M. Beneke, V. M. Braun and N. Kivel, Phys. Lett. B 443, 308 (1998) hep-ph/9809287.

[53] E.V. Shuryak, The QCD Vacuum, Hadrons and Superdense Matter, Second Edition, (World Scientific, Singapore, 2004).

[54] E. Witten, Nucl. Phys. B 160, 57 (1979).

[55] E. Witten, Nucl. Phys. B 149, 285 (1979).

[56] M. A. Shifman, Theory of Preasymptotic Effects in Weak Inclusive Decays, in Proc. Workshop on Continuous Advances in QCD, ed. A. Smilga (World Scientific, Singapore, 1994), page 249 [hep-ph/9405246]; Recent progress in the heavy quark theory, in Proc. V PASCOS Symp., March 1995, Baltimore, ed. J. Bagger (World Scientific, Singapore, 1996), page 69 [hep-ph/9505289].

[57] I. B. Khriplovich, Sov. J. Nucl. Phys. 10, 235 (1969).

[58] M. Shifman, Advanced Topics in Quantum Field Theory, (Cambridge University Press, 2012), Chapter 5.

[59] E. Witten, Phys. Lett. B 117, 324 (1982) [reprinted in Current Algebra and Anomalies, Eds. S. Treiman, R. Jackiw, B. Zumino, and E. Witten, (Princeton University Press, 1985) p. 429].

[60] D. R. T. Jones, Nucl. Phys. B 75, 531 (1974); W. E. Caswell, Phys. Rev. Lett. 33, 244 (1974). 